\title{
Une Suisse sans hépatite, une vision réaliste
}

\section{Gert Printzen}

Dr, membre du Comité central de la FMH, responsable du département eHealth - informatique et documentation médicales / Produits thérapeutiques

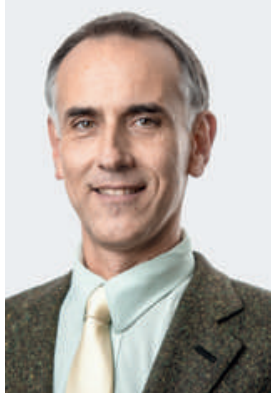

La journée mondiale de l'hépatite de l'OMS aura lieu le 28 juillet prochain. Cette date n'est pas fortuite puisqu'elle correspond au jour anniversaire de Baruch Bloomberg, qui a découvert le virus de l'hépatite B. Pour nous, l'occasion d'empoigner la question car ce n'est pas à tort si l'hépatite virale est également qualifiée d'«épidémie silencieuse». En effet, trop peu d'attention a été accordée jusqu'à présent à cette maladie qui, selon les chiffres de l'Organisation mondiale de la santé (OMS), coûte la vie à 1,4 million de personnes chaque année dans le monde [1]. Il est donc grand temps d'agir par des mesures ciblées, en Suisse aussi! Selon les estimations de l'Office fédéral de la santé publique (OFSP), entre 60000 et 80000 personnes vivraient dans notre pays avec une hépatite chronique $C$ et 20000 à 30000 avec une hépatite chronique $\mathrm{B}$ (prévention des infections transmissibles par voie sanguine, OFSP 2011 [2]). Dans la mesure où l'infection, même à un stade avancé des lésions du foie, n'est souvent accompagnée que d'un nombre restreint de symptômes, la moitié des patientes et patients concernées ignorent qu'ils sont infectés.

La vision ambitieuse de la stratégie nationale: éradiquer l'hépatite virale d'ici à 15 ans.

Le pic des infections a été atteint avant le début des années 90 pour l'hépatite $C$ (contamination par des produits sanguins ou injections de drogues par voie intraveineuse) et avant 1998 pour l'hépatite B soit avant l'entrée en vigueur de la recommandation générale de la vaccination des enfants et des jeunes entre 11 et 15 ans. Compte tenu qu'une infection à l'hépatite évolue sur plusieurs dizaines d'années, on peut s'attendre à une augmentation d'une grande partie des cas d'infections du foie entre 2020 et 2025 .

En comparaison internationale, la Suisse accuse un certain retard dans le suivi de la maladie, notamment en matière de dépistage et de surveillance, comme le révèle le rapport 2012 de l'index européen sur les hépatites [3]. Ceci est d'autant plus étonnant que nous disposons aujourd'hui de mesures de prévention et de traitement particulièrement efficaces. On relèvera l'engagement de la Suisse à mettre en œuvre une vaste stratégie de lutte contre l'infection dans le sillage de la résolution de l'OMS de 2014 et l'évolution que l'on peut qualifier de révolutionnaire en matière de traitements médicamenteux. Toutes ces innovations font de l'hépatite C une maladie guérissable [4]. Mais pour cela, les

\section{Près de la moitié des personnes atteintes d'une} hépatite l'ignorent.

médicaments aujourd'hui limités par l'OFSP doivent devenir meilleur marché et plus accessibles, la population et le corps médical doivent être (encore) mieux informés, et les groupes à risque faire l'objet de dépistage et bénéficier de traitement.

Face au danger de propagation de l'épidémie, un groupe composé d'experts, de représentantes et représentants des patients et de la FMH s'est mis à l'œuvre. Depuis janvier 2014, les travaux se concentrent autour de l'élaboration d'une stratégie nationale qui poursuit l'objectif ambitieux d'éradiquer l'hépatite dans un délai de 15 ans. Des groupes interdisciplinaires rassemblant plus de 70 experts sont déjà au travail. Les premières actions concrètes ont été définies: elles vont de la collecte de données épidémiologiques auprès des groupes concernés au développement d'une campagne de sensibilisation, en passant par la mise au point d'un programme de suivi et de surveillance, l'actualisation d'un guide de traitement ou encore une analyse du rapport coût-utilité des activités prévues. Une première phase d'implémentation s'achèvera à la fin de l'année; l'ensemble de la démarche devrait s'étendre sur 15 ans.

Manifestement, il vaut la peine de se mobiliser dès à présent, de faire des recherches sur les situations à risque - même si elles peuvent renvoyer au passé lointain des personnes concernées - et d'en faire une analyse conséquente. Ce faisant, l'acuité de la maladie et le risque d'augmentation brutale des infections du foie pourraient être un peu diminués.

Toute la motivation du groupe d'experts qui s'est constitué au fil de la procédure de développement de la stratégie et l'ensemble du travail accompli jusqu'ici à titre honorifique nous permettent d'être optimistes: une Suisse sans hépatite ne peut et ne doit pas rester un vœu pieux. Retroussons-nous les manches!

1-4 Les références se trouvent online avec des liens. 